\title{
Imaging findings in a patient with eosinophilic pneumonia \\ (Löffler's syndrome)
}

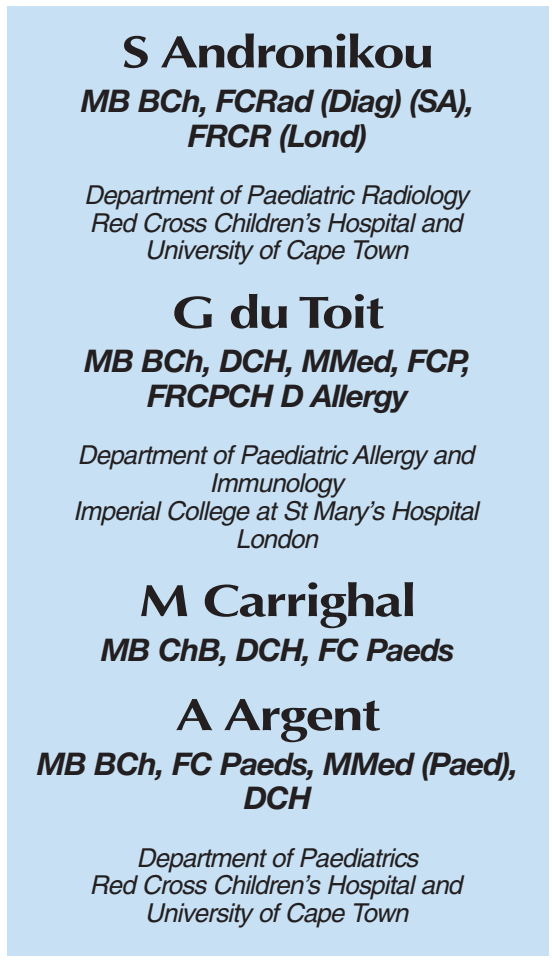

\section{Abstract}

Löffler's syndrome was initially described as a disorder characterised by transient pulmonary infiltrates accompanied by peripheral blood eosinophilia in asymptomatic or mildly ill patients. Abnormal chest radiographic findings are said to occur in $95 \%$ of patients but there are no descriptions of CT findings. There are many causes of this syndrome, but in developing countries the most common presentation remains secondary to the migratory larvae of common intestinal helminths. We present the clinical and radiological features of a boy with clearly defined Löffler's syndrome due to larval migration.

\section{Introduction}

Löffler's syndrome was initially described by the Swiss internist Wilhelm Löffler (1887 - 1972) as a clinical disorder characterised by transient pulmonary infiltrates accompanied by peripheral blood eosinophilia in asymptomatic or mildly ill patients. Migratory pulmonary infiltrates are recognised in many lung diseases $^{1}$ and the term Löffler's syndrome is used interchangeably with a myriad of other different names; these include, 'simple pulmonary eosinophilia, 'cryptogenic eosinophilic pneumonia,' 'larval pneumonitis', 'pulmonary infiltrates with eosinophilia (PIE)', and 'Löffler's pneumonia'. Dr Wilhelm Löffler also described a condition of fibroplastic parietal endocarditis with eosinophilia which further complicates the correct use of terminology.

Eosinophilic pulmonary reactions are typical of the immediate Type I allergic response, ${ }^{1}$ which may be induced by differing factors including allergic bronchopulmonary mycoses, parasitic infestations, drug reactions, eosinophilia-myalgia syndrome, Löffler's syndrome, chronic eosinophilic pneumonia, allergic granulomatosis of Churg and Strauss, hypereosinophilic syndrome, and acute eosinophilic pneumonia. ${ }^{2}$ Noninfectious causes include drug reactions (to penicillin, the sulphonamides, aminosalicylic acid, nitrofurantoin), ${ }^{3}$ smoke and dust inhalation (mouldy hay, zinc chloride, beryllium, nickel), collagen vascular diseases (polyarteritis nodosa, rheumatoid arthritis, systemic lupus erythematosis) and neoplastic disorders (Hodgkin's disease).

Infectious causes of Löffler's syndrome include protozoa, fungi, bacteria and helminths. ${ }^{1}$ In developing countries with high parasitic loads, helminthic causes predominate. The majority of worms implicated are nematodes, including Strongyloides stercoralis, Ascaris lumbricoides, Toxocara canis, Schistosoma mansoni, Paragonimus westermani, Wuchereria bancrofti, Dirofilaria immitis, Trichuris trichiura and Fasciola hepatica. ${ }^{1}$

This case study and brief review will concern itself only with Löffler's syndrome occurring as a consequence of larval migration through the lung. A spectrum of disease severity may result as a consequence of this phase in the nematode life cycle involving one or more of the following: cough with or without sputum, haemoptysis, prolonged expiration and wheezing, dyspnoea, chest pain, occasional rales, and pleural and pericardial effusion. Abnormal chest radiographic findings are said to occur in $95 \%$ of 
patients. We present the clinical and radiological findings of a young child who experienced severe respiratory compromise due to larval-induced pulmonary eosinophilia. To the best of our knowledge high-resolution computed tomography (HRCT) findings have not been described previously for Löffler's syndrome as a consequence of larval migration.

\section{Case report}

A previously well 26-month-old boy presented with fever and acute severe respiratory distress, for which he required intubation and ventilatory support. Initial investigations revealed a microcytic hypochromic anaemia (Hb $10.1 \mathrm{~g} / \mathrm{dl}, \mathrm{MCV} 73 \mathrm{fl}$ ), with an elevated WCC of $23.1 \times 10^{9} / 1$ and peripheral blood eosinophilia of $26 \%$. Erythrocyte sedimentation rate was $39 \mathrm{~mm} /$ hour. Bronchoalveolar lavage revealed $57 \%$ eosinophilia. Further investigations revealed positive Toxocara canis serology (immunoglobin G enzyme-linked immunosorbent assay > 100 units; normal $<0$ units) and Ascaris lumbricoides adults and ova were present in his stool.

Serial chest radiography demonstrated flitting bilateral patchy opacification. A HRCT was performed in the subacute phase of the disease. This demonstrated bilateral, asymmetrical, patchy, peripheral, upper and lower zone areas of interlobular septal thickening with associated areas of airspace disease in the central rather than peripheral dependent areas of the lungs (Figs $1 \mathrm{a}$ and $\mathrm{b}$ ). There was relative sparing of the anterior or nondependent areas of the lungs. The septal thickening outlining the secondary pulmonary lobules was smooth, as was the thickening around the bron- chovascular bundles. No ground glass attenuation was noted, but the HRCT was performed during the subacute phase of the illness at which time clinical and radiological resolution was occurring. The patient responded rapidly to corticosteroid and antihelminthic therapy, and at follow-up was asymptomatic with a clear chest radiograph.

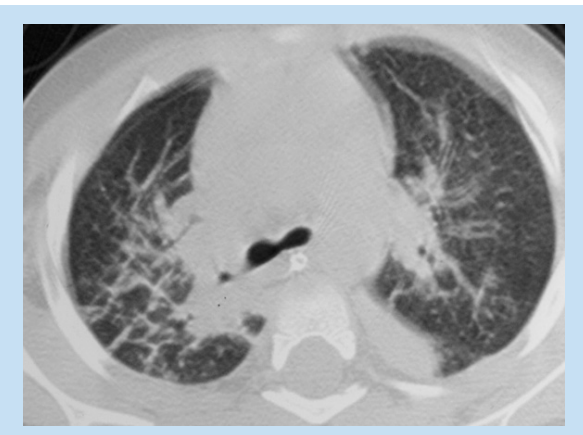

Fig. 1a. Figs .1a and b. High resolution CT scan findings of the chest. (a) Upper zone patchy areas of interlobular septal thickening, right worse than left, with associated areas of air-space disease.

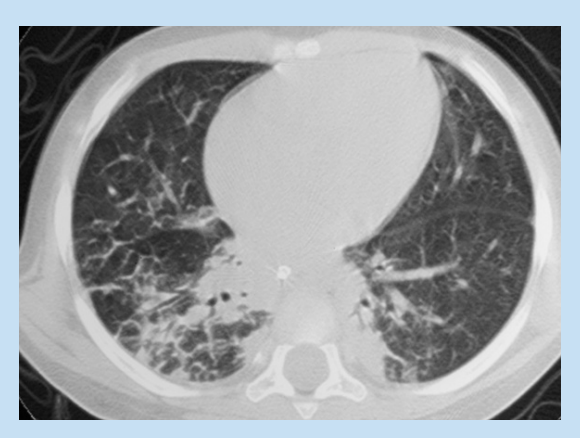

Fig. 1b. (b) Mid/ lower zone scan demonstrating patchy, predominantly peripheral and basal interlobular septal thickening outlining the secondary pulmonary lobules. There are associated central and dependent areas of dense air-space disease. The anterior portions of the lung are relatively spared.

\section{Discussion}

We report the case of a young boy who presented with life-threatening Löffler's syndrome secondary to parasitic infestation. Although our patient met many of the diagnostic criteria for acute eosinophilic pneumonia ${ }^{4}$ (Table I), a parasitic cause was unequivocally established. In making a diagnosis of Löffler's syndrome, heavy reliance is frequently placed on radiological features. Chest radiographs obtained during the migratory phase classically demonstrate fine miliary nodules or diffuse, reticulonodular interstitial areas of increased opacity. With the development of heavier infestation, bilateral, patchy air-space / alveolar areas of increased opacity and segmental or even lobar areas of opacification may develop. Serial radiographs may show migration of areas of increased opacity. Such findings are associated with several pulmonary processes, but the presence of filariform larvae in the sputum, bronchial washings, or lung biopsy specimens is diagnostic. Striking sputum and peripheral blood eosinophilia is often associated. The presence of eggs of the causative nematode, except in early infestation or with a population of purely male worms, further strengthens the diagnosis of Löffler's syndrome secondary to migratory larvae.

The HRCT findings of helminthassociated pulmonary eosinophilic pneumonitis (Löffler's syndrome) have not been reported. HRCT is generally more useful than plain radiographs in defining severity and extent of diffuse lung disease. ${ }^{5}$ HRCT findings have, however, been reported for drug-induced pulmonary eosinophilia and include bilateral, non-segmental, peripheral areas of groundglass attenuation or consolidation, indistinguishable from those seen in organising pneumonia, typically involving the upper lobes. ${ }^{3,67}$ The findings may be difficult to distinguish from adult respiratory distress syndrome, overhydration pulmonary 


\section{CASE REPORT}

\section{Table I. Proposed diagnosxtic criteria for acute eosinophilic pneumonia}

Acute febrile illness (days, rarely weeks in duration)

Hypoxaemic respiratory failure

Diffuse alveolar/mixed alveolar interstitial radiographic changes

Bronchoalveolar lavage - eosinophils $>25 \%$, or biopsy confirmation of eosinophilic lung infiltrates ${ }^{\star}$

No identifiable infection

Prompt and complete response to steroids

Failure to relapse after discontinuation of steroids

${ }^{*}$ Biopsy evidence of acute and/or organising diffuse alveolar damage with eosinophils is the most characteristic feature, but marked tissue eosinophilic infiltrate with clinical confirmation of the history is also sufficient.

oedema or atypical bacterial or viral pneumonia. ${ }^{8,9}$ Our HRCT findings correspond with the histological reports of pulmonary eosinophilia and Löffler's disease, which demonstrate thick alveolar walls and interstitial infiltration by eosinophils, lymphocytes and plasma cells. ${ }^{1,3}$ Alveolar spaces contain aggregates of histiocytes and numerous eosinophils. In contradistinction to Wegner's disease, which also has pulmonary eosinophilic infiltrates, eosinophilic pneumonia preserves the lung architecture. ${ }^{1}$ True hypersensitivity pneumonitis (usually caused in children by exposure to numerous bird antigens) shows ground-glass attenuation, but has centrilobular nodules. ${ }^{6,10}$

HRCT findings have been reported for numerous diffuse lung diseases in children including bronchiolitis obliterans, hypersensitivity pneumonitis, recurrent aspiration, Langerhans cell histiocytosis, idiopathic pulmonary haemosiderosis, pulmonary alveolar proteinosis, lymphangioleiomyomatosis, congenital lymphangiectasia, alveolar microlithiasis, pulmonary vein atresia, capillary hemangiomatosis, Gaucher's disease, NiemanPick disease, as well as for the idiopathic pneumonitides (desquamative interstitial pneumonitis (DIP), normal interstitial pneumonitis (NIP), lymphocytic interstitial pneumonitis (LIP), and chronic organising pneumonitis (COP)/ bronchiolitis obstructive organising pneumonitis (BOOP). ${ }^{5-7,10}$ The HRCT findings in our patient correspond with those reported for drug-induced pulmonary eosinophilic pneumonia, except for the absence of ground-glass attenuation. Ground-glass attenuation is a nonspecific finding in many diffuse lung diseases of childhood, usually indicating reversible or potentially reversible disease. ${ }^{6}$ The absence of this finding in our patient is probably due to the scan being performed during the subacute period of the dis- ease when acute alveolitis may have resolved spontaneously or on treatment. Follow-up HRCT may be useful in identifying chronic interstitial changes and possible fibrosis and as suggested by the HRCT findings in the sub-acute period, may also demonstrate improvement by demonstrating resolution of ground-glass attenuation.

\section{References}

1. Bedrossian CW, Greenberg SD, Williams LJ jun. Ultrastructure of the lung in Loeffler's pneumonia. Am J Med 1975; 58: 438-443.

2. Tazelaar HD, Linz LJ, Colby TV, Myers JL Limper AH. Acute eosinophilic pneumonia: histopathologic findings in nine patients. Am Respir Crit Care Med 1997; 155: 296-302.

3. Cleverley JR, Screaton NJ, Hiorns MP, Flint JD, Muller NL. Drug induced lung disease: highresolution CT and histological findings. Clin Radiol 2002; 57: 292-299.

4. Badesch DB, King TE jun, Schwarz MI. Acut eosinophilic pneumonia: a hypersensitivity phenomenon? Am Rev Respir Dis 1989; 145: 716-718.

5. Copley SJ, Coren M, Nicholson AG, Rubens MB, Bush A, Hansell DN. Diagnostic accuracy of thin-section CT and chest radiography of pediatric interstitial lung disease. $\mathrm{Am}$ Roentgenol 2000; 174: 549-554.

6. Lynch DA, Hay T, Newell JD jun, Divgi VD, Fan LL. Pediatric diffuse lung disease: diagnosis and classification using high resolution CT. Am J Roentgenol 1999; 173: 713-718.

7. Erasmus JJ, McAdams HP, Rossi SE. High-resolution CT of drug-induced lung disease. Radiol Clin North Am 2002; 40(1):61-72.

8. King MA, Pope-Harman AL, Allen JN, Christoforidis GA, Christoforidis AJ. Acute eosinophilic pneumonia: radiologic and clinical features. Radiology 1997; 203(3):715-719.

9. Cheon JE, Lee KS, Jung GS, Chung MH, Cho YD. Acute eosinophilic pneumonia: radiographic and CT findings in six patients. Am J Roentgenol 1996; 167: 1195-1199.

10. Kuhn JP, Brody AS. High resolution CT of pediatric lung disease. Radiol Clin North Am 2002; 40(1): 89-110. 